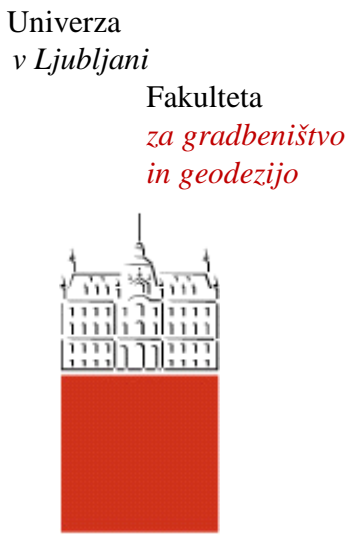

Jamova cesta 2

1000 Ljubljana, Slovenija

http://www3.fgg.uni-lj.si/

DRUGG - Digitalni repozitorij UL FGG http://drugg.fgg.uni-lj.si/

Ta članek je avtorjeva zadnja recenzirana različica, kot je bila sprejeta po opravljeni recenziji.

Prosimo, da se pri navajanju sklicujte na bibliografske podatke, kot je navedeno:
University
of Ljubljana

Faculty of

Civil and Geodetic

Engineering

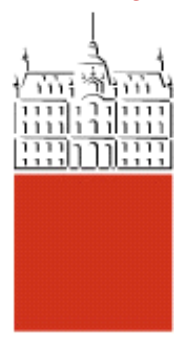

Jamova cesta 2

SI - 1000 Ljubljana, Slovenia

http://www3.fgg.uni-lj.si/en/

DRUGG - The Digital Repository http://drugg.fgg.uni-lj.si/

This version of the article is author's manuscript as accepted for publishing after the review process.

When citing, please refer to the publisher's bibliographic information as follows:

Schnabl, S., Saje, M., Turk, G., Planinc, I. 2007. Locking-free two-layer Timoshenko beam element with interlayer slip. Finite Elements in Analysis and Design 43, 9: 705-714.

DOI: 10.1016/j.finel.2007.03.002. 


\title{
Locking-free two-layer Timoshenko beam element with interlayer slip
}

\author{
S. Schnabl, M. Saje, G. Turk* and I. Planinc \\ University of Ljubljana, Faculty of Civil and Geodetic Engineering, Jamova 2, \\ SI-1115 Ljubljana, Slovenia
}

\begin{abstract}
A new locking-free strain-based finite element formulation for the numerical treatment of linear static analysis of two-layer planar composite beams with interlayer slip is proposed. In this formulation, the modified principle of virtual work is introduced as a basis for the finite element discretization. The linear kinematic equations are included into the principle by the procedure, similar to that of Lagrangian multipliers. A strain field vector remains the only unknown function to be interpolated in the finite element implementation of the principle. In contrast with some of the displacement-based and mixed finite element formulations of the composite beams with interlayer slip, the present formulation is completely locking-free. Hence, there are no shear and slip locking, poor convergence and stress oscillations in these finite elements. The generalization of the composite beam theory with the consideration of the Timoshenko beam theory for the

* Corresponding author. Tel.: +38614768 614; Fax: +386 14768629 email: gturk@fgg.uni-lj.si (G. Turk)
\end{abstract}


individual component of a composite beam represents a substantial contribution in the field of analysis of non-slender composite beams with an interlayer slip. An extension of the present formulation to the nonlinear material problems is straightforward. As only a few finite elements are needed to describe a composite beam with great precision, the new finite element formulations is perfectly suited for practical calculations.

Key-words: composite beam, interlayer slip, Timoshenko beam theory, locking, finite element method.

\section{Introduction}

Multi-layered structures have been playing an increasingly important role in different areas of engineering practice, perhaps most notably in civil, automotive, aerospace and aeronautic technology. Classical cases of such structures in civil engineering are steel-concrete composite beams in buildings and bridges, wood-concrete floor systems, coupled shear walls, concrete beams externally reinforced with laminates, sandwich beams, and many more. It is well known, that the behaviour of these structures largely depends on different materials of individual components and by the type of their connection. There exist many ways how to obtain the connection between the components. Usually, mechanical shear connectors are employed to provide a desired composite action. With the use of rigid shear connectors, a full shear connection and full composite action between the individual components can be achieved. Consequently, conventional principles of the solid beam analysis can be employed. Unfortunately, the full shear connection can hardly be materializied in practice and thus only an incomplete or partial interaction between the layers can be obtained and an 
interlayer slip often develops. In some cases it significantly effects the mechanical behaviour of composite systems.

Hence, the inclusion of the interlayer-slip effect into multi-layered beam theory is essential for optimal design and accurate representation of the actual mechanical behaviour of multi-layered structures with partial interaction between the components. Many efforts and large number of research studies have been devoted to obtaining the solution of the aforementioned problem. Early studies on beams with partial interaction between the layers were based on the assumptions of linear elastic material models and the Euler-Bernouli hypothesis of plane sections. Perhaps the first but certainly the most quoted partial action theory was developed by Newmark et al. [1]. Up to now, a number of elastic theories have been developed and presented in professional literature [2-9]. The main disadvantage of all these elastic theories and their closed form analytical solutions is that they could be obtained only for problems with simple geometry, loading and boundary conditions. Therefore, in recent years numerous investigators have refined these theories to incorporate several aspects of non-linear geometric and material behaviour [10-14] as well as torsion [15], time dependent effects [8], uplift [16] and dynamics [17]. Such complex problems are usually solved using numerical methods such as finite difference methods and finite element methods. Among all those numerical methods, the majority of researchers have employed the displacement-based $[16,18]$, force-based [19] and mixed [18-22] finite element method. It is well known, that finite element models which use low-order interpolation and a few finite elements experience so-called slip locking for high values of stiffness of the shear connection $[18,21]$. This locking is due to the inconsistent approximation of different fields governing the beam model. It is 
possible to reduce or completely eliminate locking by lowering the degree of interpolation functions for the slip or by introducing elements with larger numbers of degrees of freedom $[18,21]$.

Besides, one of the basic assumptions of all aforementioned models with partial interaction between the layers was the most commonly used Euler-Bernoulli beam theory for each individual layer, respectively. The main shortcoming of the classical Euler-Bernoulli beam theory is that no transverse shear deformation is allowed for. This implies an infinite shear stiffness of individual layer. Since, in reality, no material exists that possesses such a property, the suitability of the Euler-Bernoulli beam theory for composite beams with an interlayer slip can be questioned, especially for thick and short composite beams. In these cases, the application of the Timoshenko beam theory is indispensable for accurate prediction of the mechanical behaviour of aforementioned structures. A large number of homogeneous beam elements based on Timoshenko beam theory have appeared in the literature. To eliminate shear and membrane locking, several approaches have been proposed. Among them, the reduced or selective integration technique is the most common. An extensive list of references on locking in Timoshenko beams is not among the goals of this work. On the other hand, there seems to exist only one report on the exact solution of Timoshenko composite beam with an interlayer slip [23], and no reports on the finite element formulation of Timoshenko composite beams with the partial interaction between the layers. In the present paper, we aim to fill this gap.

The objective of this paper is two-fold. Firstly, we present a new locking-free strain-based finite element formulation for the linear static analysis of 
two-layer planar beams with interlayer slip. In this formulation, the principle of virtual work has been employed as a basis for the finite element discretization. Thus, we have proposed a modified form of the principle of virtual work by including the linear kinematic equations as constraining equations by a procedure, similar to that of Lagrangian multipliers. In this way we eliminate the displacement field vector from the principle of virtual work. As a result, the strain field vector remains the only unknown function to be approximated in the finite element implementation. This means, that only the extensional strains and pseudocurvatures of reference axis of individual layers and transverse shear strains of layer cross-sections need to be interpolated. Furthermore, the present approach uses the concept of the consistent equilibrium of constitutive and equilibrium-based stress-resultants [24] and the Galerkin type of the finite element formulation is employed [25]. In contrast with many of the aforementioned displacements-based, force-based and mixed finite element formulations of composite beams with interlayer slip, the present formulation is completely locking-free. Consequently, the ambiguous selection of consistent polynomial approximations for physically different field variables can thus be fully avoided.

The second objective of the present paper is the incorporation of the transverse shear deformation into the two-layer composite beam theory with an interlayer slip. The Timoshenko beam theory for each of the individual layer has been adopted. Since, the distribution of the transverse shear strain in the Timoshenko beam theory is assumed to be constant across the cross-section, the shear correction factor is necessary to use [26] for the appropriate representation of shear stresses through the cross-section. The 
proposed generalization of the composite beam theory with the Timoshenko beam theory is therefore an improvement in the field of analysis of non-slender (thick and short) composite beams with an interlayer slip. Finally, the common outcome of the present formulation is a family of more accurate and efficient beam finite elements for the linear static analysis of two-layer planar Timoshenko beams with an interlayer slip. Any kind of locking (shear, slip, curvature), poor convergence and stress oscillations are absent in these finite elements.

\section{Formulation of basic equations of a two-layer Timoshenko beam}

The following formulation of a two-layer planar Timoshenko composite beam with an interlayer slip is based on Reissner's [27] finite-strain beam theory, in which Bernoulli's hypothesis of plane cross-sections for each individual layer is assumed. Plane cross-sections remain planar during deformation but not necessarily perpendicular to the deformed centroidal axis of the beam. Under this assumption, the effect of a constant transverse shear strain of the individual layer can be approximately taken into account by decoupling the rotation of the cross-section from the slope of the deformed line of centroid. Accordingly, the Timoshenko [28] beam theory for each layer is applied. In addition, we assumed that displacements, strains and rotations are small and that the shapes of the cross-sections are symmetrical with respect to the plane of deformation and remain unchanged in the form and size during deformation. Layers are assumed to be continuously connected and interlayer stiffness of the connection is taken as constant. Besides, tangential slip can occur at the interface between the layers but no delamination or transverse separation between them is possible. In what follows, we briefly describe the basic equations of the two-layer Timoshenko beam. 


\subsection{Kinematic, constitutive and constraining equations}

We consider an initially straight, planar, two-layer Timoshenko beam element of undeformed length $L$, see Fig. 1 . Layers are marked by letters $a$ and $b$, respectively. The two-layer beam element is analyzed in the $(x, z)$-plane of a spatial Cartesian coordinate system with coordinates $(x, y, z)$ and unit base vectors $\mathbf{E}_{x}, \mathbf{E}_{y}, \mathbf{E}_{z}$. The reference axis of the two-layer beam element is common to both layers. It coincides with the axis $x$ and it lies in the contact plane between the layers. The geometric shape of the cross-section of each layer is assumed to be arbitrary but symmetric with respect to $(x, z)$ plane and constant along its longitudinal axis $x$. Only for the sake of clearness the cross-sections plotted in Fig.1 are rectangular. The two-layer beam element is subjected to the action of the conservative distributed load $\boldsymbol{p}=p_{x} \mathbf{E}_{x}+p_{z} \mathbf{E}_{z}$ along the span on the upper face of layer $b$. Besides, it is also subjected to generalized point forces $S_{i}^{a}$ and $S_{i}^{b}$ $(i=1,2, \ldots, 6)$ at the ends of layers $a$ and $b$.

The position vectors of material particles of the deformed configurations of layers $a$ and $b$ in the plane of deformation $(y=0)$ are defined by vector-valued functions

$$
\begin{gathered}
\mathbf{R}^{a}(x, z)=\left(x+u^{a}(x)+z \varphi^{a}(x)\right) \mathbf{E}_{x}+\left(z+w^{a}(x)\right) \mathbf{E}_{z}, \\
\mathbf{R}^{b}\left(x^{*}, z\right)=\left(x^{*}+u^{b}\left(x^{*}\right)+z \varphi^{b}\left(x^{*}\right)\right) \mathbf{E}_{x}+\left(z+w^{b}\left(x^{*}\right)\right) \mathbf{E}_{z} .
\end{gathered}
$$

In Eqs. (1) and (2), and in all further expressions, the notations $(\bullet)^{a}$ and $(\bullet)^{b}$ denote whether quantities correspond to layer $a$ or $b$. Thus, functions $u^{a}(x), w^{a}(x), \varphi^{a}(x)$ denote the longitudinal displacement along the direction of the reference axis, the transverse displacement, and the rotation of the cross-section of layer $a$ with respect to the base vectors $\mathbf{E}_{x}, \mathbf{E}_{z}$ and $\mathbf{E}_{y}$, 


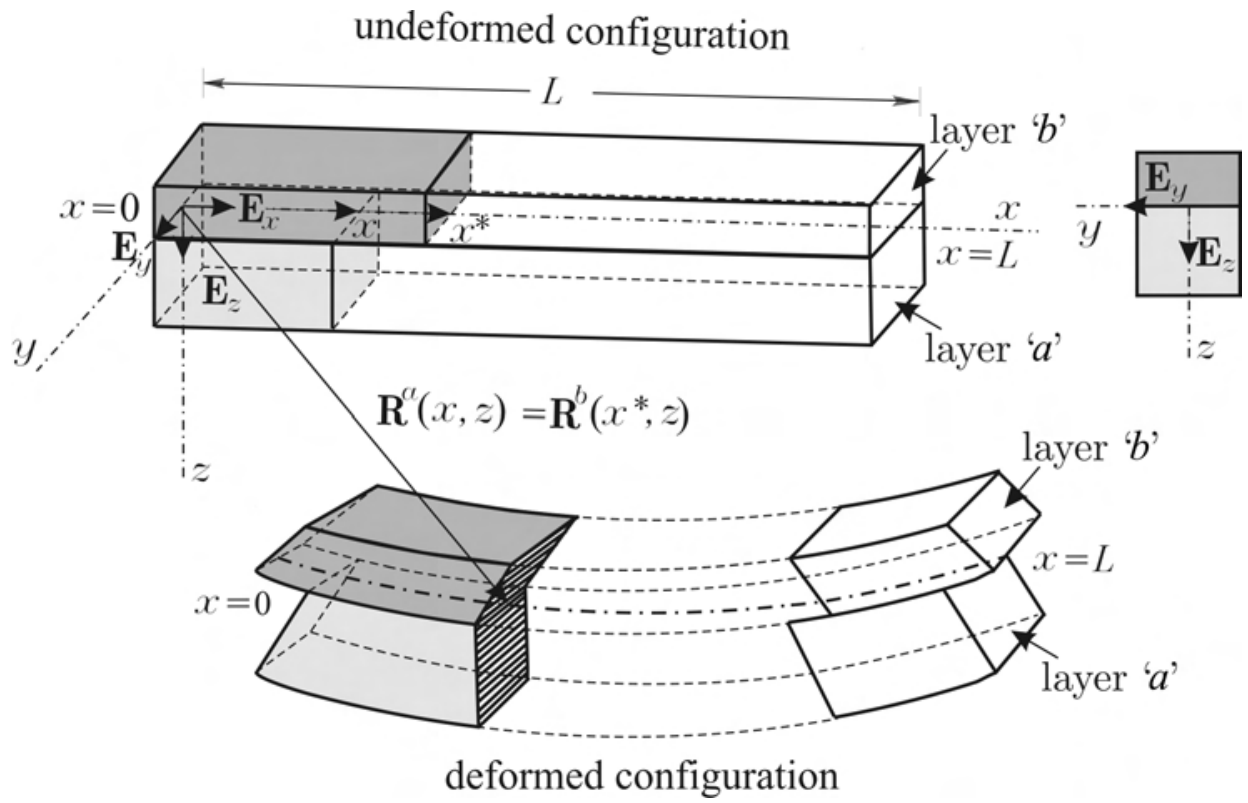

Figure 1. Undeformed and deformed configuration of the two-layer beam

respectively. Variables $u^{b}\left(x^{*}\right), w^{b}\left(x^{*}\right), \varphi^{b}\left(x^{*}\right)$ are related to layer $b$. The components of the generalized displacement vectors $\boldsymbol{u}^{a}=\left\{u^{a}(x), w^{a}(x), \varphi^{a}(x)\right\}$ and $\boldsymbol{u}^{b}=\left\{u^{b}\left(x^{*}\right), w^{b}\left(x^{*}\right), \varphi^{b}\left(x^{*}\right)\right\}$ are related to the components of the generalized strain vectors $\varepsilon^{a}=\left\{\varepsilon^{a}(x), \gamma^{a}(x), \kappa^{a}(x)\right\}$ and $\varepsilon^{b}=\left\{\varepsilon^{b}\left(x^{*}\right), \gamma^{b}\left(x^{*}\right), \kappa^{b}\left(x^{*}\right)\right\}$ by the linearized Reissner's kinematic equations $[9,12]$ :

$$
\begin{aligned}
u^{a \prime}(x)-\varepsilon^{a}(x)=0, & u^{b \prime\left(x^{*}\right)}-\varepsilon^{b}\left(x^{*}\right)=0, \\
w^{a \prime(x)}+\varphi^{a}(x)-\gamma^{a}(x)=0, & w^{b \prime\left(x^{*}\right)}+\varphi^{b}\left(x^{*}\right)-\gamma^{b}\left(x^{*}\right)=0, \\
\varphi^{a \prime(x)}-\kappa^{a}(x)=0, & \varphi^{b \prime\left(x^{*}\right)}-\kappa^{b}\left(x^{*}\right)=0 .
\end{aligned}
$$

In Eqs.(3-5) the prime $\left({ }^{\prime}\right)$ denotes the first derivative with respect to either $x$ or $x^{*}$, whereas functions $\varepsilon, \gamma, \kappa$ mark the extensional strain, the transverse shear strain and the pseudocurvature of the individual layer, respectively. Conjugate to these strains we have stress resultants 
$\boldsymbol{\sigma}^{a}=\left\{\mathcal{N}^{a}(x), \mathcal{Q}^{a}(x), \mathcal{M}^{a}(x)\right\}$ and $\boldsymbol{\sigma}^{b}=\left\{\mathcal{N}^{b}\left(x^{*}\right), \mathcal{Q}^{b}\left(x^{*}\right), \mathcal{M}^{b}\left(x^{*}\right)\right\}$ for the equilibrium axial forces $\mathcal{N}^{a}(x), \mathcal{N}^{b}\left(x^{*}\right)$, the transverse shear forces $\mathcal{Q}^{a}(x), \mathcal{Q}^{b}\left(x^{*}\right)$ and the bending moments $\mathcal{M}^{a}(x), \mathcal{M}^{b}\left(x^{*}\right)$ of the individual layer. In order to relate the equilibrium axial and shear forces, and the equilibrium moments to material models of layers, we introduce a set of constitutive equations which assures the balance of the equilibrium and constitutive cross-sectional resultants. For linear elastic material, the constitutive functions $\mathcal{N}_{\mathrm{C}}^{a}(x), \mathcal{N}_{\mathrm{C}}^{b}\left(x^{*}\right), \mathcal{Q}_{\mathrm{C}}^{a}(x), \mathcal{Q}_{\mathrm{C}}^{b}\left(x^{*}\right), \mathcal{M}_{\mathrm{C}}^{a}(x), \mathcal{M}_{\mathrm{C}}^{b}\left(x^{*}\right)$ can be given in terms of the components of the generalized strain vectors $\varepsilon^{a}$ and $\varepsilon^{b}$. Thus the constitutive equations of layers are defined by equations

$$
\begin{gathered}
\mathcal{N}^{a}(x)=\mathcal{N}_{\mathrm{C}}^{a}\left(x, \varepsilon^{a}(x), \kappa^{a}(x)\right)=E^{a} A^{a} \varepsilon^{a}(x)+E^{a} S^{a} \kappa^{a}(x), \\
\mathcal{N}^{b}\left(x^{*}\right)=\mathcal{N}_{\mathrm{C}}^{b}\left(x^{*}, \varepsilon^{b}\left(x^{*}\right), \kappa^{b}\left(x^{*}\right)\right)=E^{b} A^{b} \varepsilon^{b}\left(x^{*}\right)+E^{b} S^{b} \kappa^{b}\left(x^{*}\right), \\
\mathcal{Q}^{a}(x)=\mathcal{Q}_{\mathrm{C}}^{a}\left(x, \gamma^{a}(x)\right)=G^{a} A_{\mathrm{S}}^{a} \gamma^{a}(x), \\
\mathcal{Q}^{b}\left(x^{*}\right)=\mathcal{Q}_{\mathrm{C}}^{b}\left(x^{*}, \gamma^{b}\left(x^{*}\right)\right)=G^{b} A_{\mathrm{S}}^{b} \gamma^{b}\left(x^{*}\right), \\
\mathcal{M}^{a}(x)=\mathcal{M}_{\mathrm{C}}^{a}\left(x, \varepsilon^{a}(x), \kappa^{a}(x)\right)=E^{a} S^{a} \varepsilon^{a}(x)+E^{a} J^{a} \kappa^{a}(x), \\
\mathcal{M}^{b}\left(x^{*}\right)=\mathcal{M}_{\mathrm{C}}^{b}\left(x^{*}, \varepsilon^{b}\left(x^{*}\right), \kappa^{b}\left(x^{*}\right)\right)=E^{b} S^{b} \varepsilon^{b}\left(x^{*}\right)+E^{b} J^{b} \kappa^{b}\left(x^{*}\right),
\end{gathered}
$$

in which $A^{a}, A^{b}$ are the areas of cross-sections, $E^{a}, E^{b}$ are the elastic modulus, $S^{a}, S^{b}$ are the static moments of area and $J^{a}, J^{b}$ are the cross-sectional moments of inertia of layers $a$ and $b$ with respect to the reference axis of the whole cross-section of the two-layer beam element. In addition, the $A_{\mathrm{S}}^{a}$ and $A_{\mathrm{S}}^{b}$ represent the areas of the shear cross-sections [26].

Once the layers are connected together, the upper layer is constrained to follow the deformation of lower layer, and vice versa. As already stated, the layers can slip along each other, but their transverse separation or 
penetration is not allowed. This fact is expressed by the kinematic-constraint requirement

$$
\mathbf{R}^{a}(x, z)=\mathbf{R}^{b}\left(x^{*}, z\right),
$$

where $x \in \mathcal{I}^{a}, x^{*} \in \mathcal{I}^{b}$ are undeformed coordinates of two distinct particles of layers $a$ and $b$ which are in the deformed state in contact, and thus their vector-valued functions $\mathbf{R}^{a}(x, z)$ and $\mathbf{R}^{b}\left(x^{*}, z\right)$ coincide (see Fig. 1). Eq. (12) can be rewritten in a more convenient component form:

$$
\begin{aligned}
x+u^{a}(x) & =x^{*}+u^{b}\left(x^{*}\right), \\
w^{a}(x) & =w^{b}\left(x^{*}\right) .
\end{aligned}
$$

The relative displacements (slip) that occurs between the two particles which coincide in the undeformed configuration is denoted by $\Delta$, and is in the case of geometrically linear beam theory simply given by

$$
\Delta(x)=u^{a}(x)-u^{b}(x)=u^{a}(0)-u^{b}(0)+\int_{0}^{x}\left(\varepsilon^{a}(x)-\varepsilon^{b}(x)\right) \mathrm{d} \xi .
$$

In the present paper, the linear constitutive law of the bond slip between the layers is assumed.

$$
p_{t}=K \Delta
$$

where $K$ represents the interlayer slip modulus. For a detailed explanation of the constraining equations, a reader is directed to the Refs. [9, 11-13, 23].

Assuming strains, displacements, rotations and slips to be small quantities, the Eqs. (3-5) can be simplified using the following two assumptions (see, e.g. [12]): (i) $d x \approx d x^{*}$; (ii) vertical deflections of the reference axis of individual layers are equal $w^{a}(x)=w^{b}\left(x^{*}\right)=w(x)$ and $\mathcal{I}^{a} \approx \mathcal{I}^{b}=[0, L]$. Thus, all quantities of layer $b$ are equal at $x$ and $x^{*}$, e.g. $u^{b}\left(x^{*}\right)=u^{b}(x)$. Due to the last two assumptions, the arguments in the following equations can be 
omitted. This implies that a simplified version of the Eqs. (3-5) reads:

$$
\begin{aligned}
u^{a \prime}-\varepsilon^{a}=0, & u^{b \prime}-\varepsilon^{b}=0, \\
w^{\prime}+\varphi^{a}-\gamma^{a}=0, & w^{\prime}+\varphi^{b}-\gamma^{b}=0, \\
\varphi^{a \prime}-\kappa^{a}=0, & \varphi^{b \prime}-\kappa^{b}=0 .
\end{aligned}
$$

Since the constraining equations define the conditions that assemble an individual layer into a layered composite beam, the Eqs. (17-19) are not independent of each other. The application of Eqs. (18-19) to the first and second derivative of Eq. (14) with respect to $x$, gives modified Eqs. (18-19) by which the rotations and pseudocurvatures of layers are constrained to each other. According to the above simplification, the modified kinematic equations of the two-layer Timoshenko beam read

$$
\begin{gathered}
u^{a \prime}-\varepsilon^{a}=0, \\
u^{b \prime}-\varepsilon^{b}=0, \\
w^{a \prime}+\varphi^{a}-\gamma^{a}=0, \\
\varphi^{a \prime}-\kappa^{a}=0, \\
\varphi^{b}-\varphi^{a}+\gamma^{a}-\gamma^{b}=0, \\
\kappa^{b}-\kappa^{a}+\gamma^{a \prime}-\gamma^{b \prime}=0 .
\end{gathered}
$$

2.2 The modified principle of virtual work and its finite element formulation

The principle of virtual work states that the difference of virtual works of 
internal and external forces is zero

$$
\begin{gathered}
\delta W=\delta W^{a}+\delta W^{b}=\int_{0}^{L}\left(\mathcal{N}^{a} \delta \varepsilon^{a}+\mathcal{Q}^{a} \delta \gamma^{a}+\mathcal{M}^{a} \delta \kappa^{a}\right) \mathrm{d} x+ \\
+\int_{0}^{L}\left(\mathcal{N}^{b} \delta \varepsilon^{b}+\mathcal{Q}^{b} \delta \gamma^{b}+\mathcal{M}^{b} \delta \kappa^{b}\right) \mathrm{d} x+\int_{0}^{L}\left(p_{t} \delta u^{a}-p_{n} \delta w\right) \mathrm{d} x- \\
-\int_{0}^{L}\left(\left(p_{t}+p_{x}\right) \delta u^{b}+\left(p_{n}-p_{z}\right) \delta w\right) \mathrm{d} x- \\
-S_{1}^{a} \delta u^{a}(0)-S_{2}^{a} \delta w(0)-S_{3}^{a} \delta \varphi^{a}(0)-S_{4}^{a} \delta u^{a}(L)-S_{5}^{a} \delta w(L)-S_{6}^{a} \delta \varphi^{a}(L)- \\
-S_{1}^{b} \delta u^{b}(0)-S_{2}^{b} \delta w(0)-S_{3}^{b} \delta \varphi^{b}(0)-S_{4}^{b} \delta u^{b}(L)-S_{5}^{b} \delta w(L)-S_{6}^{b} \delta \varphi^{b}(L)=0 .
\end{gathered}
$$

Here, $\delta u^{a}, \delta u^{b}, \delta w$ are virtual displacements, $\delta \varepsilon^{a}, \delta \varepsilon^{b}, \delta \gamma^{a}, \delta \gamma^{b}, \delta \kappa^{a}, \delta \kappa^{b}$ are virtual strains of the reference axis of the composite beam; $\delta u^{a}(0), \delta u^{a}(L)$, $\delta w(0)$, etc., denote the virtual boundary displacements, whereas $p_{n}$ represents the normal interlayer contact traction. The principle given in Eq. (26) has been derived on the basis of the assumption that the kinematic and strain variables as well as their variations are constraint by the kinematic and constitutive Eqs. (20-25) and (6-11). Hence, only six among the eleven functions $u^{a}, u^{b}, w, \varphi^{a}, \varphi^{b}, \varepsilon^{a}, \varepsilon^{b}, \gamma^{a}, \gamma^{b}, \kappa^{a}$ and $\kappa^{b}$ are mutually independent. These constraints are released if the $\mathrm{Hu}$-Washizu functional is introduced with Eqs. (20-25) as being a set of constraining equations of the functional. The Eq. (20-25) are scalarly multiplied by arbitrary, independent, and at least once differentiable Lagrangian multipliers $\Lambda_{i}$. The scalar products of the multipliers and the constraining equations are integrated along the length $L$ and varied with respect to displacements, strains and Lagrangian multitpliers. The terms that contain first derivatives of displacements and strains are partially integrated. After adding the obtained expressions to Eq. (26), the strain-based principle of virtual work called a modified principle of 
virtual work is derived $[12,25]$

$$
\begin{gathered}
\delta W_{\mathrm{mod}}=\int_{0}^{L}\left(\left(\mathcal{N}_{\mathrm{C}}^{a}-\Lambda_{1}\right) \delta \varepsilon^{a}+\left(\mathcal{N}_{\mathrm{C}}^{b}-\Lambda_{2}\right) \delta \varepsilon^{b}+\left(\mathcal{Q}_{\mathrm{C}}^{a}-\Lambda_{3}+\mathcal{M}_{\mathrm{C}}^{b \prime}\right) \delta \gamma^{a}\right. \\
\left.+\left(\mathcal{Q}_{\mathrm{C}}^{b}-\mathcal{M}_{\mathrm{C}}^{b \prime}\right) \delta \gamma^{b}+\left(\mathcal{M}_{\mathrm{C}}^{a}+\mathcal{M}_{\mathrm{C}}^{b}-\Lambda_{4}\right) \delta \kappa^{a}\right) \mathrm{d} x+\left(u^{a}(L)-u^{a}(0)-\int_{0}^{L} \varepsilon^{a} \mathrm{~d} x\right) \delta \Lambda_{1}(0) \\
+\left(u^{b}(L)-u^{b}(0)-\int_{0}^{L} \varepsilon^{b} \mathrm{~d} x\right) \delta \Lambda_{2}(0)+\left(w(L)-w(0)-\int_{0}^{L}\left(\gamma^{a}-\varphi^{a}\right) \mathrm{d} x\right) \delta \Lambda_{3}(0) \\
+\left(\varphi^{a}(L)-\varphi^{a}(0)-\int_{0}^{L} \kappa^{a} \mathrm{~d} x\right) \delta \Lambda_{4}(0) \\
-\left(S_{1}^{a}+\Lambda_{1}(0)\right) \delta u^{a}(0)-\left(S_{1}^{b}+\Lambda_{2}(0)\right) \delta u^{b}(0)-\left(S_{2}^{a}+S_{2}^{b}+\Lambda_{3}(0)\right) \delta w(0) \\
-\left(S_{3}^{a}+S_{3}^{b}+\Lambda_{4}(0)\right) \delta \varphi^{a}(0)-\left(S_{4}^{a}-\Lambda_{1}(L)\right) \delta u^{a}(L)-\left(S_{4}^{b}-\Lambda_{2}(L)\right) \delta u^{b}(L) \\
-\left(S_{5}^{a}+S_{5}^{b}-\Lambda_{3}(L)\right) \delta w(L)-\left(S_{6}^{a}+S_{6}^{b}-\Lambda_{4}(L)\right) \delta \varphi^{a}(L)=0
\end{gathered}
$$

This strain-based formulation offers a number of advantages, such as a consistent cross-sectional equilibrium and a derivation of locking-free strain-based finite elements. The functional (27) stated above represents the starting point of the strain-based Galerkin-type of the finite element discretization. The only unknown functions defining the principle (27) are the strain variables-the axial strains $\varepsilon^{a}, \varepsilon^{b}$, the transverse shear strains $\gamma^{a}$, $\gamma^{b}$ and the pseudocurvature $\kappa^{a}$. Notice, that the displacements, rotations, forces and moments are included only through their boundary values. Thus, functions $\varepsilon^{a}, \varepsilon^{b}, \gamma^{a}, \gamma^{b}, \kappa^{a}$ and twelve parameters $\Lambda_{1}(0), \Lambda_{2}(0), \Lambda_{3}(0), \Lambda_{4}(0), u^{a}(0), u^{a}(L), u^{b}(0), u^{b}(L)$, $w(0), w(L), \varphi^{a}(0), \varphi^{a}(L)$ where $\Lambda_{1}, \Lambda_{2}, \Lambda_{3}, \Lambda_{4}$ represent the Euler-Lagrange multipliers (in this case, the forces and moments in global coordinate system), fully describe the functional (27). In the finite element implementation of the principle, we need to interpolate five strain functions 
$\varepsilon^{a}(x), \varepsilon^{b}(x), \gamma^{a}(x), \gamma^{b}(x), \kappa^{a}(x)$ and their variations. In this way, the reference axis of the two-layer Timoshenko beam is divided into finite elements.

Within each element, the strain functions and their variations are interpolated. For the interpolation of the strain functions, the Lagrangian polynomials $P_{n}\left(n=1,2, \ldots, N_{I}\right)$ of degree $N_{I}-1$ are employed. Additionally, it is assumed that the variations of strain functions are approximated by the Dirac $\delta$-function. The definition of Dirac-delta function, collocation method and the fundamental lemma of the calculus of variation used to derive the discrete system of Euler-Lagrange equations can be found in [29]. The selection of the collocation points $x_{i}\left(i=1,2, \ldots, N_{K}\right)$ is crucial in obtaining a well conditioned system of equations and a convergent solution. Thus, the interpolation of the unknowns takes the form

$$
\begin{array}{ll}
\varepsilon^{a}(x) \doteq \sum_{n=1}^{N_{I}} P_{n}(x) \varepsilon_{n}^{a}, & \delta \varepsilon^{a}(x) \doteq \delta\left(x-x_{i}\right), \\
\varepsilon^{b}(x) \doteq \sum_{n=1}^{N_{I}} P_{n}(x) \varepsilon_{n}^{b}, & \delta \varepsilon^{b}(x) \doteq \delta\left(x-x_{i}\right), \\
\gamma^{a}(x) \doteq \sum_{n=1}^{N_{I}} P_{n}(x) \gamma_{n}^{a}, & \delta \gamma^{a}(x) \doteq \delta\left(x-x_{i}\right), \\
\gamma^{b}(x) \doteq \sum_{n=1}^{N_{I}} P_{n}(x) \gamma_{n}^{b}, & \delta \gamma^{b}(x) \doteq \delta\left(x-x_{i}\right), \\
\kappa^{a}(x) \doteq \sum_{n=1}^{N_{I}} P_{n}(x) \kappa_{n}^{a}, & \delta \kappa^{a}(x) \doteq \delta\left(x-x_{i}\right) .
\end{array}
$$

Discrete values $\varepsilon_{n}^{a}, \varepsilon_{n}^{b}, \gamma_{n}^{a},, \gamma_{n}^{b}, \kappa_{n}^{a}$ represent the nodal values of the interpolated functions. Thus, for the construction of the finite-element model of the two-layer Timoshenko beam with an interlayer slip the Petrov-Galerkin collocation method is used. For the sake of the simplicity, we assume that the interpolation and collocation points within the element 
coincide: $N=N_{I}=N_{K}$. Assuming further that equilibrium equations

$$
\begin{array}{ll}
\mathcal{N}^{a \prime}-p_{t}=0, & \mathcal{N}^{b \prime}+p_{t}+p_{x}=0, \\
\mathcal{Q}^{a \prime}+p_{n}=0, & \mathcal{Q}^{b \prime}+p_{z}-p_{n}=0, \\
\mathcal{M}^{a \prime}+Q^{a}=0, & \mathcal{M}^{b \prime}-Q^{b}=0 .
\end{array}
$$

are identically satisfied, the boundary forces $\Lambda_{1}(L), \Lambda_{2}(L), \Lambda_{3}(L)$, ans the boundary moment $\Lambda_{4}(L)$ in Eq. (27) can easily be expressed only by $\Lambda_{1}(0), \Lambda_{2}(0), \Lambda_{3}(0), \Lambda_{4}(0)$ and $p_{z}, p_{x}, p_{x}$ and $p_{z}$. Insertion of Eqs. (28-32) into the variational principle (27) and using the fundamental lema of the calculus of variation yield the discrete system of Euler-Lagrange equations of the principle:

$$
\begin{gathered}
f_{i}=\left.\left(\mathcal{N}_{\mathrm{C}}^{a}-\Lambda_{1}\right)\right|_{x=x_{i}}=0 \quad i=1, \ldots, N \\
f_{N+j}=\left.\left(\mathcal{N}_{\mathrm{C}}^{b}-\Lambda_{2}\right)\right|_{x=x_{j}}=0 \quad j=1, \ldots, N \\
f_{2 N+k}=\left.\left(\mathcal{Q}_{\mathrm{C}}^{a}-\Lambda_{3}+\mathcal{M}_{\mathrm{C}}^{b \prime}\right)\right|_{x=x_{k}}=0 \quad k=1, \ldots, N \\
f_{3 N+l}=\left.\left(\mathcal{Q}_{\mathrm{C}}^{b}-\mathcal{M}_{\mathrm{C}}^{b \prime}\right)\right|_{x=x_{l}}=0 \quad l=1, \ldots, N \\
f_{4 N+m}=\left.\left(\mathcal{M}_{\mathrm{C}}^{a}+\mathcal{M}_{\mathrm{C}}^{b}-\Lambda_{4}\right)\right|_{x=x_{m}}=0 \\
f_{5 N+1}=u^{a}(L)-u^{a}(0)-\int_{0}^{L} \varepsilon^{a} \mathrm{~d} x=0 \\
f_{5 N+2}=u^{b}(L)-u^{b}(0)-\int_{0}^{L} \varepsilon^{b} \mathrm{~d} x=0 \\
f_{5 N+3}=w(L)-w(0)-\int_{0}^{L}\left(\gamma^{a}-\varphi^{a}\right) \mathrm{d} x=0 \\
f_{5 N+4}=\varphi^{a}(L)-\varphi^{a}(0)-\int_{0}^{L} \kappa^{a} \mathrm{~d} x=0 \\
f_{5 N+5}=S_{1}^{a}+\Lambda_{1}(0)=0
\end{gathered}
$$




$$
\begin{gathered}
f_{5 N+6}=S_{1}^{b}+\Lambda_{2}(0)=0 \\
f_{5 N+7}=S_{2}^{a}+S_{2}^{b}+\Lambda_{3}(0)=0 \\
f_{5 N+8}=S_{3}^{a}+S_{3}^{b}+\Lambda_{4}(0)=0 \\
f_{5 N+9}=S_{4}^{a}-\Lambda_{1}(0)-\int_{0}^{L} p_{t} \mathrm{~d} x=0 \\
f_{5 N+10}=S_{4}^{b}-\Lambda_{2}(0)+\int_{0}^{L}\left(p_{x}+p_{t}\right) \mathrm{d} x=0 \\
f_{5 N+11}=S_{5}^{a}+S_{5}^{b}-\Lambda_{3}(0)+\int_{0}^{L} p_{z} \mathrm{~d} x=0 \\
f_{5 N+12}=S_{6}^{a}+S_{6}^{b}-\Lambda_{4}(0)-\int_{0}^{L} \Lambda_{3} \mathrm{~d} x=0
\end{gathered}
$$

For a given load factor, $\lambda$, Eqs. (34-50) constitute a system of $5 N+12$ linear algebraic equations for $5 N+12$ unknowns. There are $5 N+4$ internal degrees of freedom $\varepsilon_{n}^{a}, \varepsilon_{n}^{b}, \gamma_{n}^{a}, \gamma_{n}^{b}, \kappa_{n}^{a}, \Lambda_{1}(0), \Lambda_{2}(0), \Lambda_{3}(0), \Lambda_{4}(0)$, and eight external degrees of freedom, i.e., nodal displacement and rotations $u^{a}(0)$, $u^{a}(L), u^{b}(0), u^{b}(L), w(0), w(L), \varphi^{a}(0), \varphi^{a}(L)$ of the finite element. The internal degrees of freedom are eliminated from the structural assemblage by the static condensation at the element level. The condensed global tangent stiffness matrix and the condensed residual force vector of the structure in then assembled in a classical way. For the solution of the equations a standard method for solutions of linear system can be employed. Notice, that for non-singular solution of Eqs. (34-50) at least one longitudinal boundary displacement, belonging either to layer $a$ or $b$, must be prescribed.

\section{Numerical examples}

The following examples demonstrate high accuracy and excellent performance of the proposed familly of locking-free two-layer Timoshenko beam finite elements. The purposes of the discussion presented herein are the following: (i) to check the convergence properties and locking (slip and 
shear) behaviour of presented finite elements; and (ii) to briefly investigate the influence of shear rigidity on the mechanical behaviour of continuous two-layer Timoshenko composite beam with interlayer slip.

For this purpose, we consider two simple, but indicative examples: (1) a simply supported two-layer Timoshenko composite beam with length $L$; and (2) a continuous two-layer Timoshenko composite beam over two spans. In both cases the beams are subjected to conservative distributed load of intensity $p_{z}$. The elastic properties $E^{a}, E^{b}, G^{a}$ and $G^{a}$, cross-sectional areas $A^{a}, A^{b}$ and all other material and geometric parameters are shown in Fig. 2 and Fig. 6. A shear-correction factor $k_{\mathrm{S}}$ for a rectangular cross-section is taken to be $5 / 6$ [26].

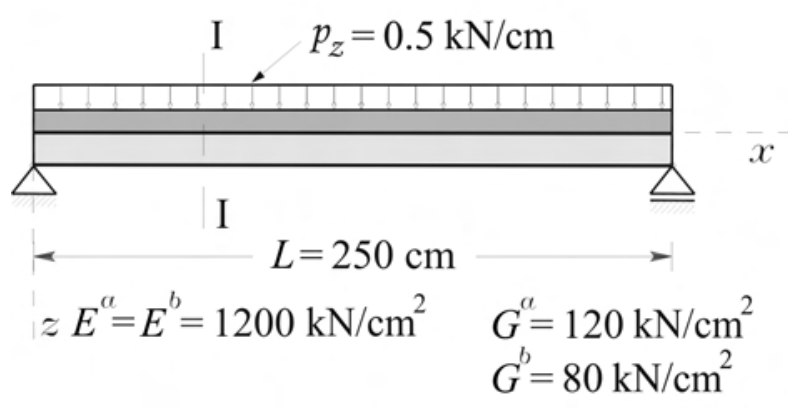

\section{Section I-I:}

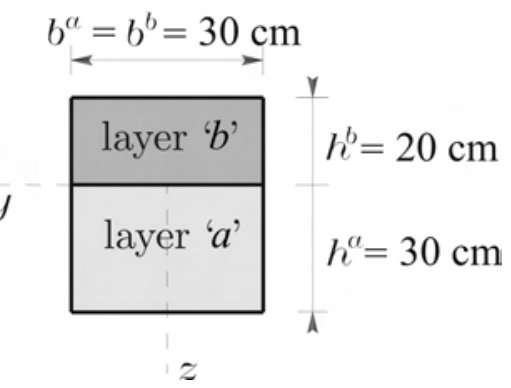

Figure 2. The descriptive geometric, material and loading data of the simply supported two-layer Timoshenko beam

In order to validate and confirm the accuracy and the convergence performance of the elements developed in the earlier section, the numerical results such as mid-point vertical deflections and interlayer slips at the edge of the beam are evaluated for different types of elements and compared to the corresponding reference solution, which is taken to be the solution by 1000 finite elements with degree of interpolation polynomials, here termed by $E_{0}$. This way we analyze the influence of the degree of interpolation 
functions (Lagrange interpolation polynomials), the number of elements and the location of collocation points $\left\{x_{1}, \ldots, x_{n}\right\}$ on the numerical results of the Timoshenko two-layer beam. As discussed in Section 2, the set of collocation points can generally be prescribed arbitrarily. Thus, the results for mid-point vertical deflection $w^{a}(L / 2)$, interlayer slip at the left edge of the beam $\Delta(0)$, and $L^{2}$-norm of a balance function of equilibrium and constitutive bending moments $\left\|\mathcal{M}_{\mathrm{C}}-\mathcal{M}\right\|_{2}$, as a function of number of elements, the collocation points and the order of interpolation polynomials are displayed in Tables 1-4 for collocation points distributed equidistantly including boundary nodes (E), by Lobatto (L), Gaussian (G), and Chebyshev (C) quadrature points. Since the type and the degree of numerical integration are always chosen such that numerical integration is exact, their influence on the results is not investigated.

As mentioned above, a variety of finite elements and element meshes have been applied. The simply supported two-layer Timoshenko beam has been modeled by $1,2,4,8,10,20,50,100$ and 1000 elements with $0,1^{\text {st }}, 2^{\text {nd }}, \ldots$, $5^{\text {th }}$ degree interpolation polynomials, here termed by $E_{0}, E_{1}, E_{2}, \ldots, E_{5}$. By employing only one element $E_{0}$ and $E_{1}$, the relative error of the computed mid-point vertical deflection and interlayer slip is significant; by increasing the number of elements $E_{0}$ and $E_{1}$, the error decreases but, the convergence to the reference solution is relatively slow. On the other hand, the error is much smaller and the convergence is much faster, if, the degree of interpolation polynomials is increased. As observed from Tables 1-4, 2 elements $E_{4}, 4$ elements $E_{3}, 8$ or 10 elements $E_{2}, 1000$ elements $E_{0}$, or only one element $E_{5}$ give the mid-point vertical deflection and interlayer slip which are accurate to 6 digits. Note that good agreement between the 
various collocation schemes is observed. Different choices of the location of the collocation points give nearly identical results for elements $E_{2}-E_{5}$, but not for elements $E_{1}$. In this case, the Gaussian $(\mathrm{G})$ collocation scheme indicates to be the most appropriate. It can also be observed from Tables 1-4 that the norm $\left\|\mathcal{M}_{\mathrm{C}}-\mathcal{M}\right\|_{2}$ decreases uniformly by increasing the number of elements and the order of interpolation functions. We may then conclude that the present finite element solution is convergent to the reference one.

Another advantage of the present finite elements is, that they are completely locking-free. It is well known, that the inherent disadvantage of some finite element models is the so-called locking. In the case of Timoshenko composite beam finite elements with an interlayer slip, the typical locking problems are shear and slip locking. The latter strongly depends on the connection stiffness $[18,21]$. This is a problem of particular interest especially in the case of high connection stiffnesses, where the slip oscillations may occur $[18,21]$. In order to show that the present finite elements are slip-locking-free, the distribution of interlayer slip along the span of a simply supported beam is shown for low (Fig. 3) and high (Fig. 4) connection stiffness. It can be observed, that in both cases, the finite elements posses neither slip-locking nor slip oscillations.

Only the results for one and two elements with low order interpolation polynomials are shown (e.g. $1 E_{1} / G$ means one element $E_{1}$ with the collocation points chosen to be distributed accordingly to the Gaussian integration scheme). For other cases not shown in Figs. 3-4 the results practically coincide with the reference distribution of interlayer slip, which would not have been the case, if slip-locking would be present. 


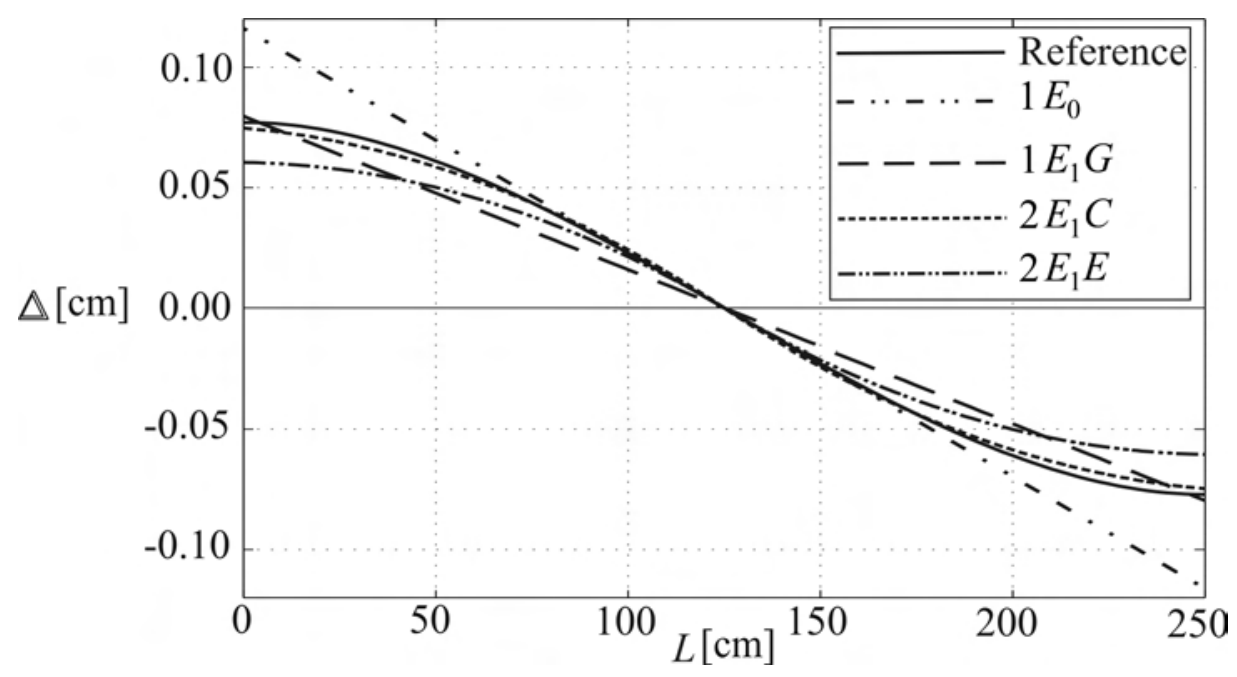

Figure 3. The distribution of interlayer slip over the span of a simply supported beam for $K=0.243 \mathrm{kN} / \mathrm{cm}^{2}$

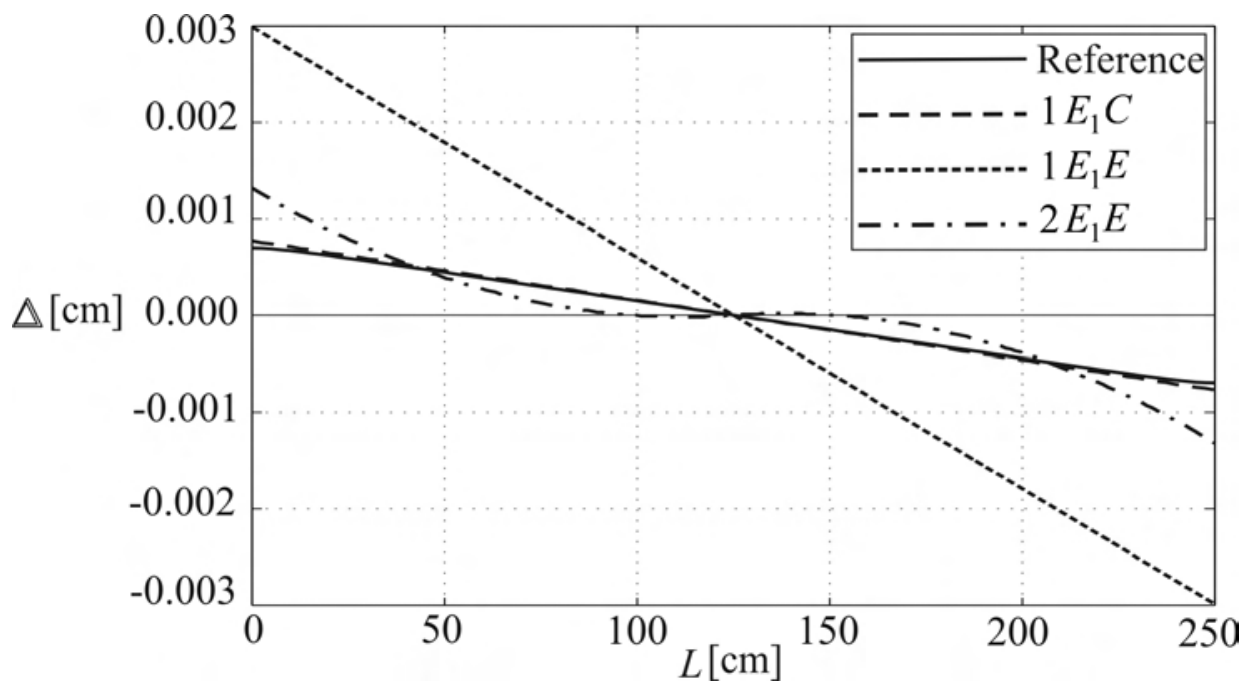

Figure 4. The distribution of interlayer slip over the span of a simply supported beam for $K=2430 \mathrm{kN} / \mathrm{cm}^{2}$

In order to demonstrate that the present finite elements are also free of shear locking, the vertical deflections $\left(w_{T}\right)$ of the two-layer Timoshenko composite 
beam with the partial interlayer interaction are compared to the vertical deflections $\left(w_{B}\right)$ obtained by the Euler-Bernoulli composite beam model with the same partial interlayer interaction, for different $L / h$ ratios and different number of finite elements with different degrees of interpolation polynomials and different collocation points schemes. It can be observed from Fig. 5, that in the limiting case where the beam becomes very slender, the results of the Timoshenko two-layer beam converge to the Euler-Bernoulli solution of the two-layer beam with an interlayer slip which is not the case for finite elements which exibite shear locking. Thus, we may conclude, that the present finite elements of the two-layer Timoshenko composite beam are shear-locking-free.

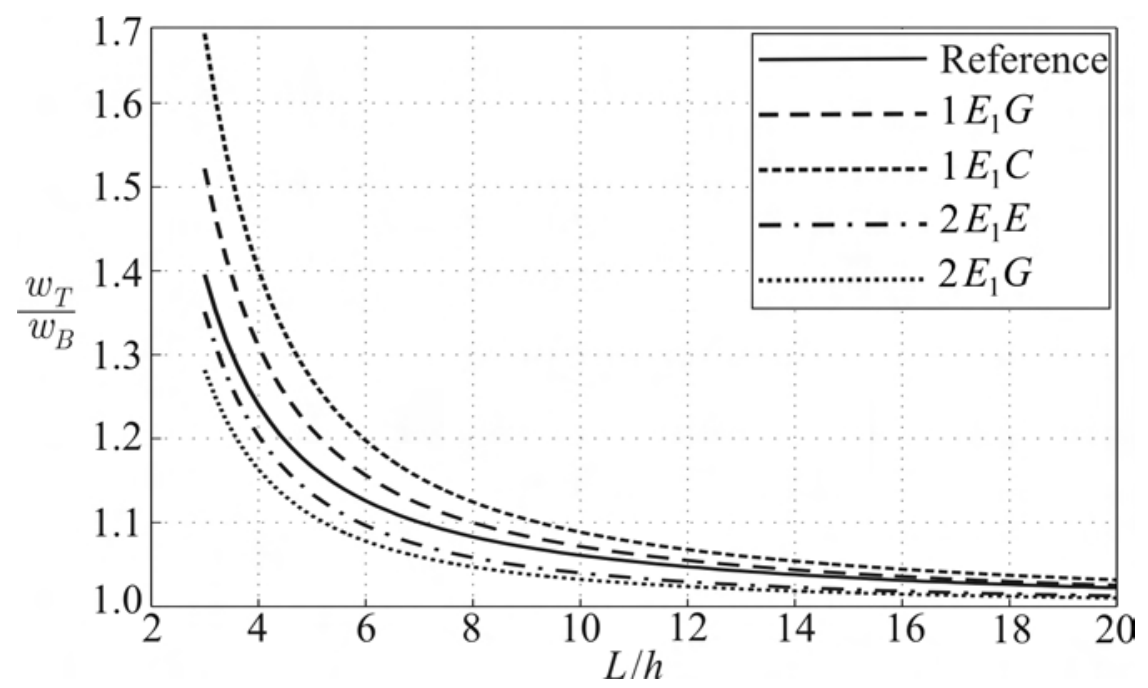

Figure 5 . The influence of $L / h$ ratios on vertical deflections of a simply supported two-layer Timoshenko composite beam 
The next example will demonstrate the application of the present locking-free strain-besed finite element method to stress-strain analysis of more complex structures. We consider a continuous asymmetric Timoshenko composite beam over two spans with the interlayer slip modulus $K=0.243 \mathrm{kN} / \mathrm{m}^{2}$. The descriptive geometric, material and loading data are described in Fig. 6.
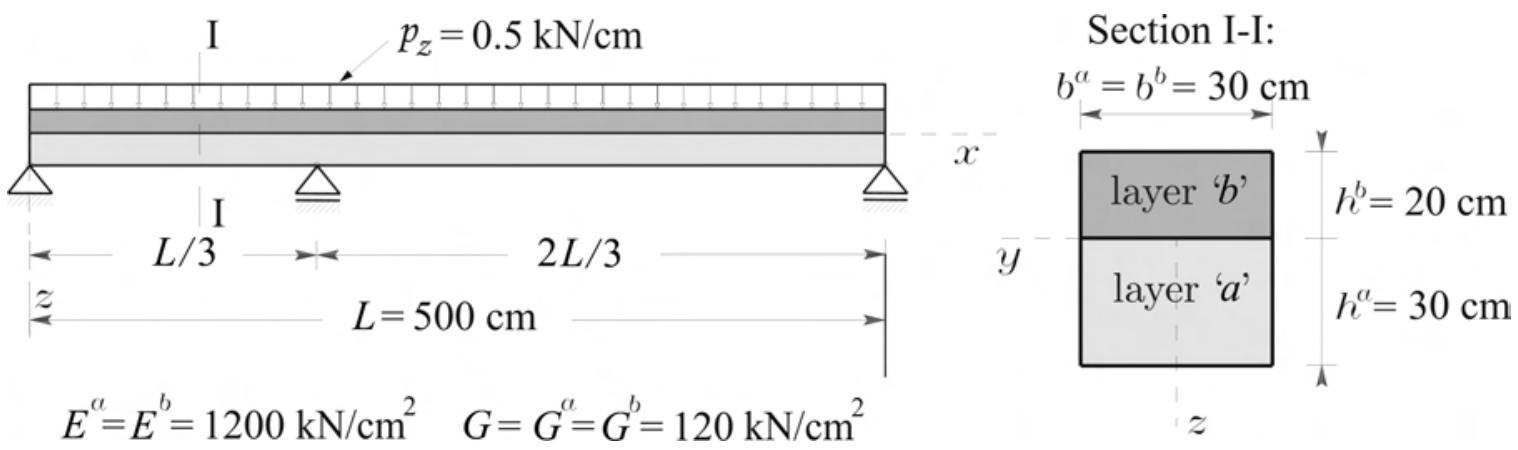

Figure 6. The descriptive geometric, material and loading data of a continuous two-layer Timoshenko composite beam over two spans

A parametric study has been conducted to briefly asses the influence of shear moduli of the layers on the values of various static and kinematic quantities. Figs. 7 and 8 show only the graphs of interlayer slip $\Delta$ and vertical deflection $w$ as a function of shear modulus $G=G^{a}=G^{b}$. The beam has been modeled by ten elements $E_{4}$ with the equidistantly distributed collocation points $(E)$. It is obvious from Figs. 7-8, that the shear modulus $G$ has an important influence on static and kinematic quantities. Observe, that slip $\Delta$ over the left span is smaller, while slip over the right span is, in contrast, higher for higher shear moduli. On the other hand, the vertical deflection $w$ over the left span is higher, while vertical deflection over the right span is smaller for higher shear moduli. For a detailed analysis of the influence of shear moduli on the mechanical behaviour of two-layer Timoshenko beams with interlayer 
slip the reader is refered to [23].

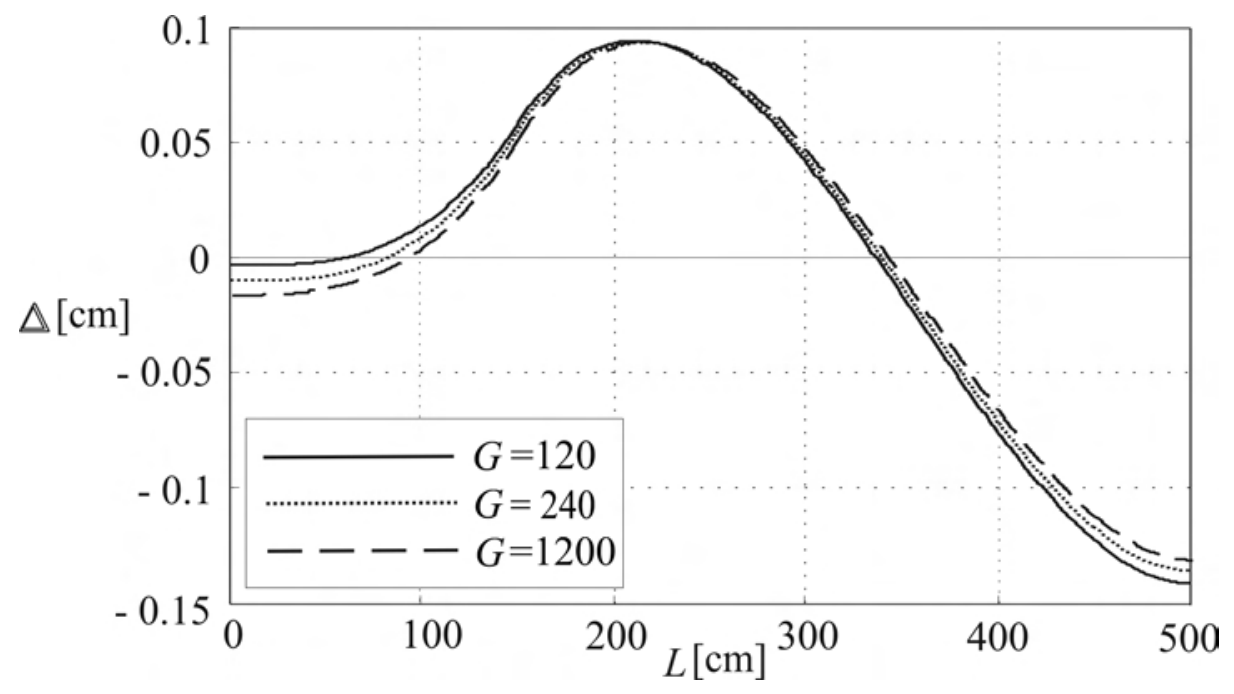

Figure 7. Distribution of $\Delta$ along the span as a function of different values of shear moduli, $G=G^{a}=G^{b}$

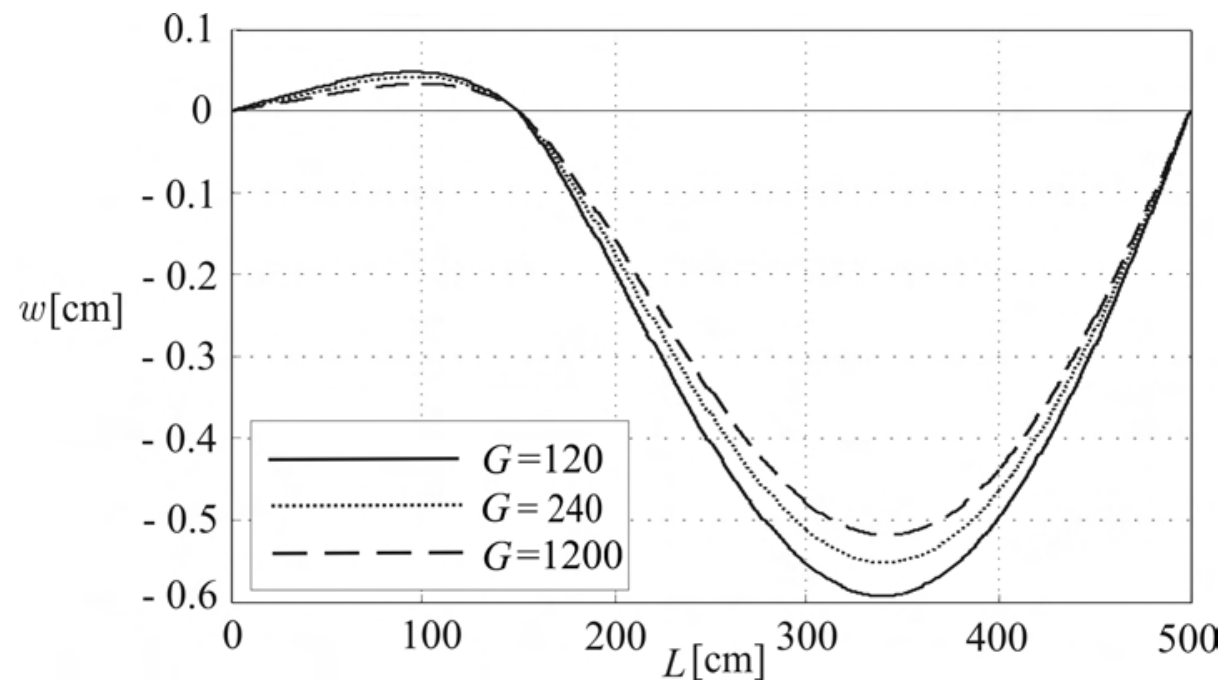

Figure 8. Distribution of $w$ along the span as a function of different values of shear moduli, $G=G^{a}=G^{b}$

\section{Conclusions}


A new locking-free strain-based finite element formulation for the numerical treatment of linear static analysis of two-layer planar composite beams with interlayer slip has been proposed. In this formulation, the modified principle of virtual work has been employed as a basis for the finite element discretization. The linear kinematic equations have been included into the principle by the procedure, similar to that of Lagrangian multipliers. A strain field vector remains the only unknown function to be approximated in the finite element implementation of the principle. As a result, in contrast with many of the displacement-based and mixed finite element formulations of the composite beams with an interlayer slip, the present formulation is completely locking-free. The generalization of the composite beam theory with the inclusion of the Timoshenko beam theory for the individual layer of composite beam represents a substantial contribution in the field of analysis of non-slender composite beams with an interlayer slip. The main outcome of the present formulation is a family of efficient beam finite elements for the linear static analysis of two-layer planar Timoshenko beams with an interlayer slip. An extension of the present formulation to nonlinear material problems is straightforward. There are no locking (shear and slip), poor convergence or stress oscillations in these finite elements. As only a few finite elements are needed to describe a composite beam of a frame with great precision, the new finite element formulations is perfectly suited for practical calculations.

\section{Acknowledgment}

The work of S. Schnabl was financially supported by the Ministry of Education, Science and Sport of the Republic of Slovenia under contract 3311-02-831625. The support is gratefully acknowledged. 


\section{Bibliography}

[1] Newmark NM, Siest CP, Viest CP. Test and analysis of composite beams with incomplete interaction. Proc Soc Exp Stress Anal 1951; 9(1):75-92.

[2] Goodman JR, Popov EP. Layered beam systems with inter-layer slip. J Struct Div ASCE 1968; 94(11): 2535-2547.

[3] Goodman JR, Popov EP. Layered wood systems with inter-layer slip. Wood Science 1969; 1(3): 148-158.

[4] Girhammar UA, Gopu VKA. Composite beam-columns with inter-layer slip-exact analysis. J Struct Eng ASCE 1993; 199(4): 1265-1282.

[5] Jasim NA. Computation of deflections for continuous composite beams with partial interaction. Proc Inst Civil Eng, Struc and Build 1997; 122: $347-354$.

[6] Jasim NA, Ali AAM. Deflections of composite beams with partial shear connection. Struct. Engineer 1997; 75: 58-61.

[7] Ranzi G, Bradford MA, Uy B. A general method of analysis of composite beams with partial interaction. Steel and Composite Struct 2003; 3(3): $169-184$.

[8] Ranzi G, Bradford MA. Analytical solution for the time-dependent behaviour of composite beams with partial interaction. Int J Solids Struct 2006; 43: 3770-3793.

[9] Schnabl S, Planinc I, Saje M, Čas B, Turk G. An analytical model of layered continuous beams with partial interaction. Struct Eng and Mech 
2006; 22(3): 263-278.

[10] Ayoub A. A two-field mixed variational principle for partially connected composite beams. Finite Elem Anal Des 2001; 37: 929-959.

[11] Čas B, Saje M, Planinc I. Nonlinear finite element analysis of composite planar frames with inter-layer slip. Comput Struct 2004; 82: 1901-1912.

[12] Čas B, Bratina S, Saje M, Planinc I, Non-linear analysis of composite steel-concrete beams with incomplete interaction. Steel and Comp Struct 2004; 4(6): 489-507.

[13] Čas B. Non-linear analysis of composite beams with inter-layer slip.

Ph.D. Thesis (in Slovene), Faculty of Civil and Geodetic Engineering, University of Ljubljana, Slovenia, 2004.

[14] Fabbrocino G, Manfredi G, Cosenza E. Modelling of continuous steel-concrete composite beams: computational aspects. Comput Struct 2002; 80: 2241-2251.

[15] Dall'Asta A. Composite beams with weak shear connection. Int J Solids Struct 2001; 38: 5605-5624.

[16] Ranzi G, Ansourian P, Gara F, Leoni G, Dezi L. Displacement-Based Formulations for Composite Beams with Longitudinal Slip and vertical Uplift. Research Report No. R837, The University of Sydney, January, 2005.

[17] Girhammar UA, Pan D. Dynamic analysis of composite members with interlayer slip. Int J Solids Struct 1993; 33(6): 797-823.

[18] Dall'Asta A, Zona A. Three-field mixed formulation for the non-linear 
analysis of composite beams with deformable shear connection. Finite Elem Anal Des 2004; 40: 425-448.

[19] Salari MR, Spacone E. Analysis of steel-concrete composite frames with bond-slip. J Struct Eng ASCE 2001; 127(11): 1241-1250.

[20] Dall'Asta A, Zona A. Non-linear analysis of composite beams by a displacement approach. Comput Struct 2002; 80: 2217-2228.

[21] Dall'Asta A, Zona A. Slip locking in finite elements for composite beams with deformable shear connection. Finite Elem Anal Des 2004; 40: $1907-1930$.

[22] Ayoub A, Filippou F. Mixed formulation of nonlinear steel-concrete composite beam element. J Struct Eng ASCE 2000; 126(3): 371-381.

[23] Schnabl S, Saje M, Turk G, Planinc I. Analytical solution of two-layer beam taking into account interlayer slip and shear deformation. J Struct Eng ASCE, accepted for publication.

[24] Vratanar B, Saje M. A consistent equilibrium in a cross-section of an elastic-plastic beam. Int J Solids Struct 1999; 36: 311-337.

[25] Planinc I, Saje M, Čas B. On the local stability condition in the planar beam finite element. J Struct Eng Mech 2001; 12(5): 507-526.

[26] Cowper GR. The shear coefficient in Timoshenko's beam theory. J Appl Mech 1966; 33(2): 335-340.

[27] Reissner E. On one-dimensional finite-strain beam theory: The plane problem. J Appl Mech Phys (ZAMP) 1972; 23: 795-804. 
[28] Timoshenko SP. On the correction for shear of the differential equation for transverse vibrations of prismatic bars. Phil Mag 1921; Series 6, 41(245): $744-746$.

[29] Reddy JN. Applied functional analysis and variational methods in engineering, McGraw-Hill Book Company, Singapore, 1986. 


\section{List of figures}

Fig. 1: Undeformed and deformed configuration of the two-layer beam.

Fig. 2: The descriptive geometric, material and loading data of the simply supported twolayer Timoshenko beam.

Fig. 3: The distribution of interlayer slip over the span of a simply supported beam for $K=0.243 \mathrm{kN} / \mathrm{cm}^{2}$.

Fig. 4: The distribution of interlayer slip over the span of a simply supported beam for $K=2430 \mathrm{kN} / \mathrm{cm}^{2}$.

Fig. 5: The influence of $L / h$ ratios on vertical deflections of a simply supported two-layer Timoshenko beam.

Fig. 6: The descriptive geometric, material and loading data of a continuous two-layer Timoshenko composite beam over two spans

Fig. 7: Distribution of $\Delta$ along the span as a function of different values of shear moduli,

$$
G=G^{a}=G^{b}
$$

Fig. 8: Distribution of $w$ along the span as a function of different values of shear moduli,

$$
G=G^{a}=G^{b}
$$


Table 1

The comparison of numerical results for one element with the reference solution.

\begin{tabular}{|c|c|c|c|c|c|}
\hline$n_{e}$ & d.o.i. & type & $w^{a}(L / 2)$ & $\Delta(0)$ & $\left\|\mathcal{M}_{\mathrm{C}}-\mathcal{M}\right\|_{2}$ \\
\hline 1 & 0 & $\mathrm{E} / \mathrm{L} / \mathrm{G} / \mathrm{C}$ & 0.290643 & 0.116257 & $1.78210 \cdot 10^{4}$ \\
\hline \multirow[t]{3}{*}{1} & 1 & $\mathrm{E} / \mathrm{L}$ & 0.033613 & 0.002988 & $2.83842 \cdot 10^{4}$ \\
\hline & & $\mathrm{G}$ & 0.225855 & 0.079659 & $1.18826 \cdot 10^{4}$ \\
\hline & & $\mathrm{C}$ & 0.178063 & 0.060640 & $1.34222 \cdot 10^{4}$ \\
\hline \multirow[t]{3}{*}{1} & 2 & $\mathrm{E} / \mathrm{L}$ & 0.270938 & 0.077249 & $1.93548 \cdot 10^{-10}$ \\
\hline & & G & 0.270972 & 0.077287 & $4.32163 \cdot 10^{-10}$ \\
\hline & & $\mathrm{C}$ & 0.270959 & 0.077273 & $3.18158 \cdot 10^{-10}$ \\
\hline \multirow[t]{4}{*}{1} & 3 & $\mathrm{E}$ & 0.270988 & 0.077271 & $3.56716 \cdot 10^{-10}$ \\
\hline & & $\mathrm{L}$ & 0.271028 & 0.077289 & $2.29257 \cdot 10^{-10}$ \\
\hline & & G & 0.270996 & 0.077288 & $3.25371 \cdot 10^{-10}$ \\
\hline & & $\mathrm{C}$ & 0.271005 & 0.077286 & $3.31368 \cdot 10^{-10}$ \\
\hline \multirow[t]{4}{*}{1} & 4 & $\mathrm{E}$ & 0.270993 & 0.077293 & $1.87862 \cdot 10^{-10}$ \\
\hline & & $\mathrm{L}$ & 0.270993 & 0.077293 & $2.89523 \cdot 10^{-10}$ \\
\hline & & G & 0.270993 & 0.077293 & $2.34103 \cdot 10^{-10}$ \\
\hline & & $\mathrm{C}$ & 0.270993 & 0.077540 & $3.30434 \cdot 10^{-10}$ \\
\hline \multirow[t]{4}{*}{1} & 5 & $\mathrm{E}$ & 0.271026 & 0.077293 & $1.42962 \cdot 10^{-10}$ \\
\hline & & $\mathrm{L}$ & 0.271026 & 0.077293 & $2.29257 \cdot 10^{-10}$ \\
\hline & & G & 0.271026 & 0.077293 & $1.23514 \cdot 10^{-10}$ \\
\hline & & $\mathrm{C}$ & 0.271026 & 0.077293 & $2.82211 \cdot 10^{-10}$ \\
\hline \multicolumn{3}{|c|}{ Reference solution } & 0.271026 & 0.077293 & $\mathbf{0}$ \\
\hline
\end{tabular}

$n_{e}$-number of elements, d.o.i.--degree of interpolation

E-equidistant, L-Lobatto, G-Gaussian, C-Chebyshev 
Table 2

The comparison of numerical results with the reference solution.

\begin{tabular}{|c|c|c|c|c|c|}
\hline$n_{e}$ & d.o.i. & type & $w^{a}(L / 2)$ & $\Delta(0)$ & $\left\|\mathcal{M}_{\mathrm{C}}-\mathcal{M}\right\|_{2}$ \\
\hline 2 & 0 & $\mathrm{E} / \mathrm{L} / \mathrm{G} / \mathrm{C}$ & 0.246928 & 0.086513 & $1.72959 \cdot 10^{4}$ \\
\hline \multirow[t]{3}{*}{2} & 1 & $\mathrm{E} / \mathrm{L}$ & 0.215860 & 0.060491 & $1.00353 \cdot 10^{4}$ \\
\hline & & G & 0.263520 & 0.079511 & $4.20113 \cdot 10^{3}$ \\
\hline & & $\mathrm{C}$ & 0.251622 & 0.074765 & $4.74548 \cdot 10^{3}$ \\
\hline \multirow[t]{3}{*}{2} & 2 & $\mathrm{E} / \mathrm{L}$ & 0.271029 & 0.077288 & $2.87028 \cdot 10^{-10}$ \\
\hline & & $\mathrm{G}$ & 0.271014 & 0.077290 & $2.31795 \cdot 10^{-10}$ \\
\hline & & $\mathrm{C}$ & 0.271020 & 0.077290 & $4.21815 \cdot 10^{-10}$ \\
\hline \multirow[t]{4}{*}{2} & 3 & $\mathrm{E}$ & 0.271031 & 0.077290 & $4.54423 \cdot 10^{-10}$ \\
\hline & & $\mathrm{L}$ & 0.271033 & 0.077291 & $3.35126 \cdot 10^{-10}$ \\
\hline & & G & 0.271034 & 0.077291 & $3.24815 \cdot 10^{-10}$ \\
\hline & & $\mathrm{C}$ & 0.271033 & 0.077291 & $2.86409 \cdot 10^{-10}$ \\
\hline \multirow[t]{4}{*}{2} & 4 & $\mathrm{E}$ & 0.271026 & 0.077293 & $3.96382 \cdot 10^{-10}$ \\
\hline & & $\mathrm{L}$ & 0.271026 & 0.077293 & $5.54296 \cdot 10^{-10}$ \\
\hline & & G & 0.271026 & 0.077293 & $4.08983 \cdot 10^{-10}$ \\
\hline & & $\mathrm{C}$ & 0.271026 & 0.077293 & $6.22722 \cdot 10^{-10}$ \\
\hline 4 & 0 & $\mathrm{E} / \mathrm{L} / \mathrm{G} / \mathrm{C}$ & 0.264388 & 0.079038 & $1.2927 \cdot 10^{4}$ \\
\hline \multirow[t]{3}{*}{4} & 1 & $\mathrm{E} / \mathrm{L}$ & 0.251631 & 0.074756 & $3.54803 \cdot 10^{3}$ \\
\hline & & G & 0.263518 & 0.079501 & $1.48532 \cdot 10^{3}$ \\
\hline & & $\mathrm{C}$ & 0.260547 & 0.078315 & $1.67778 \cdot 10^{3}$ \\
\hline \multicolumn{2}{|c|}{ Reference solution } & & 0.271026 & 0.077293 & $\mathbf{0}$ \\
\hline
\end{tabular}

$n_{e}{ }^{-}$number of elements, d.o.i.-degree of interpolation

E-equidistant, L-Lobatto, G-Gaussian, C-Chebyshev 
Table 3

The comparison of numerical results with the reference solution.

\begin{tabular}{|c|c|c|c|c|c|}
\hline$n_{e}$ & d.o.i. & type & $w^{a}(L / 2)$ & $\Delta(0)$ & $\left\|\mathcal{M}_{\mathrm{C}}-\mathcal{M}\right\|_{2}$ \\
\hline \multirow[t]{3}{*}{4} & 2 & $\mathrm{E} / \mathrm{L}$ & 0.271023 & 0.077291 & $5.11367 \cdot 10^{-10}$ \\
\hline & & G & 0.271021 & 0.077291 & $7.05474 \cdot 10^{-10}$ \\
\hline & & $\mathrm{C}$ & 0.271022 & 0.077291 & $6.60154 \cdot 10^{-10}$ \\
\hline \multirow[t]{4}{*}{4} & 3 & $\mathrm{E}$ & 0.271026 & 0.077293 & $1.03876 \cdot 10^{-10}$ \\
\hline & & $\mathrm{L}$ & 0.271026 & 0.077293 & $3.65277 \cdot 10^{-10}$ \\
\hline & & $G$ & 0.271026 & 0.077293 & $4.67220 \cdot 10^{-10}$ \\
\hline & & $\mathrm{C}$ & 0.271026 & 0.077293 & $4.07509 \cdot 10^{-10}$ \\
\hline 8 & 0 & $\mathrm{E} / \mathrm{L} / \mathrm{G} / \mathrm{C}$ & 0.268728 & 0.077167 & $9.26026 \cdot 10^{3}$ \\
\hline \multirow[t]{3}{*}{8} & 1 & $\mathrm{E} / \mathrm{L}$ & 0.260549 & 0.078315 & $1.25442 \cdot 10^{3}$ \\
\hline & & G & 0.263519 & 0.079501 & $5.25141 \cdot 10^{2}$ \\
\hline & & $\mathrm{C}$ & 0.262776 & 0.079204 & $5.93185 \cdot 10^{2}$ \\
\hline \multirow[t]{3}{*}{8} & 2 & $\mathrm{E} / \mathrm{L}$ & 0.271026 & 0.077293 & $6.92304 \cdot 10^{-10}$ \\
\hline & & G & 0.271026 & 0.077293 & $7.45907 \cdot 10^{-10}$ \\
\hline & & $\mathrm{C}$ & 0.271026 & 0.077293 & $1.01180 \cdot 10^{-10}$ \\
\hline 10 & 0 & $\mathrm{E} / \mathrm{L} / \mathrm{G} / \mathrm{C}$ & 0.269248 & 0.0769432 & $8.29534 \cdot 10^{3}$ \\
\hline \multirow[t]{3}{*}{10} & 1 & $\mathrm{E} / \mathrm{L}$ & 0.261619 & 0.078742 & $8.9758 \cdot 10^{2}$ \\
\hline & & G & 0.263519 & 0.079501 & $3.7576 \cdot 10^{2}$ \\
\hline & & $\mathrm{C}$ & 0.263044 & 0.079312 & $4.2445 \cdot 10^{2}$ \\
\hline \multirow[t]{3}{*}{10} & 2 & $\mathrm{E} / \mathrm{L}$ & 0.271026 & 0.077293 & $4.14354 \cdot 10^{-10}$ \\
\hline & & G & 0.271026 & 0.077293 & $6.45604 \cdot 10^{-10}$ \\
\hline & & $\mathrm{C}$ & 0.271026 & 0.077293 & $1.01005 \cdot 10^{-10}$ \\
\hline \multicolumn{2}{|c|}{ Reference solution } & & 0.271026 & 0.077293 & 0 \\
\hline
\end{tabular}

$n_{e}$-number of elements, d.o.i.-degree of interpolation

E-equidistant, L-Lobatto, G-Gaussian, C-Chebyshev 
Table 4

The comparison of numerical results for constant interpolation with the reference solution.

\begin{tabular}{lccccl}
\hline$n_{e}$ & d.o.i. & type & $w^{a}(L / 2)$ & $\Delta(0)$ & $\left\|\mathcal{M}_{\mathrm{C}}-\mathcal{M}\right\|_{2}$ \\
\hline 20 & 0 & $\mathrm{E} / \mathrm{L} / \mathrm{G} / \mathrm{C}$ & 0.269942 & 0.077187 & $5.87765 \cdot 10^{2}$ \\
\hline 50 & 0 & $\mathrm{E} / \mathrm{L} / \mathrm{G} / \mathrm{C}$ & 0.270457 & 0.077278 & $3.71945 \cdot 10^{-2}$ \\
\hline 100 & 0 & $\mathrm{E} / \mathrm{L} / \mathrm{G} / \mathrm{C}$ & 0.271013 & 0.077286 & $2.31448 \cdot 10^{-7}$ \\
\hline 1000 & 0 & $\mathrm{E} / \mathrm{L} / \mathrm{G} / \mathrm{C}$ & $\mathbf{0 . 2 7 1} \mathbf{0 2 6}$ & $\mathbf{0 . 0 7 7} \mathbf{2 9 3}$ & $1.31255 \cdot 10^{-10}$ \\
\hline Reference solution & & $\mathbf{0 . 2 7 1} \mathbf{0 2 6}$ & $\mathbf{0 . 0 7 7} \mathbf{2 9 3}$ & $\mathbf{0}$ \\
\hline
\end{tabular}

$n_{e}-$ number of elements, d.o.i.--degree of interpolation

E-equidistant, L-Lobatto, G-Gaussian, C-Chebyshev 